\title{
Journal of real-time image processing: first issue of volume 12
}

\author{
Nasser Kehtarnavaz ${ }^{1} \cdot$ Matthias F. Carlsohn $^{2}$
}

Published online: 13 May 2016

(C) Springer-Verlag Berlin Heidelberg 2016

\begin{abstract}
Although we are half way through the 11th publication year of Journal of Real-Time Image Processing, the four issues of volume 11 have already appeared this year and this issue constitutes the first issue of volume 12. This is due to the fact that this year in 2016, two volumes or a total of eight issues are being published. The additional volume 12 is arranged for the purpose of significantly reducing the backlog of accepted papers that have already appeared as online-first articles but are waiting to appear in print.
\end{abstract}

An editorial board meeting of JRTIP was recently held in Brussels in April 2016 which took place during the SPIE Conference on Real-Time Image and Video Processing as part of SPIE Photonics Europe. We thank our colleagues who made the meeting possible by their presence in Brussels despite the difficult circumstances that existed. A picture of the board members and the journal editor attending the meeting appears in Fig. 1.

After general information on the merger between the publishing companies Springer and Nature with no impact on the organizational aspects of JRTIP and a brief overview of the JRTIP publishing report, the following major topics were discussed during the meeting: (1) improving the turnaround time of the review process, (2) problems related to copyright aspects and self-archiving of preprint articles, (3) creating critical mass for special issues in light of the increase in the number of pages per issue, and (4) the

Nasser Kehtarnavaz

kehtar@utdallas.edu

Matthias F. Carlsohn

Matthias.Carlsohn@t-online.de

University of Texas at Dallas, Richardson, TX, USA

2 Engineering and Consultancy for Computer Vision and Image Communication, Bremen, Germany handling of significant increase that has occurred in the number of manuscript submissions.

To address the difficulties of finding reviewers who provide timely and quality reviews, it was recommended to select potential reviewers from the list of references of submitted manuscripts, to follow-up reviews by proactive engagement with reviewers and timely reminders, and if necessary to provide reviews by the handling associate editor when timeliness becomes significantly affected. The substantial increase in the number of submissions in 2016 compared to 2015 is partly due to the fact that many manuscripts whose scope does not match the real-time thrust of the journal are being submitted by authors who fail to take the real-time theme of the journal into consideration. To address copyright issues, it is important to note the Springer's Open Access publishing policy including author rights under Springer's copyright statement and its self-archiving policy as outlined at the link http://www.springer.com/gp/open-access/authors-rights/ self-archiving-policy/2124.

Another topic discussed at the meeting among the associate editors present, who all had conducted special issues previously, was the impact of their special issues due to the coverage of hot topics in real-time image and video processing, generating a trigger for awareness of hot topics, and serving as a catalyst toward recruiting motivated associate editors to the editorial board. Considering that the number of pages per issue has now grown to 200 pages, it is important for future special issue proposals to generate a critical mass of articles that are relevant to the real-time thrust of the journal on a focussed topic of interest. It would be greatly beneficial if colleagues who wish to be guest editors of JRTIP first contact potential authors in advance before proposing a special issue to make sure a critical mass of articles can be offered as a special issue. 


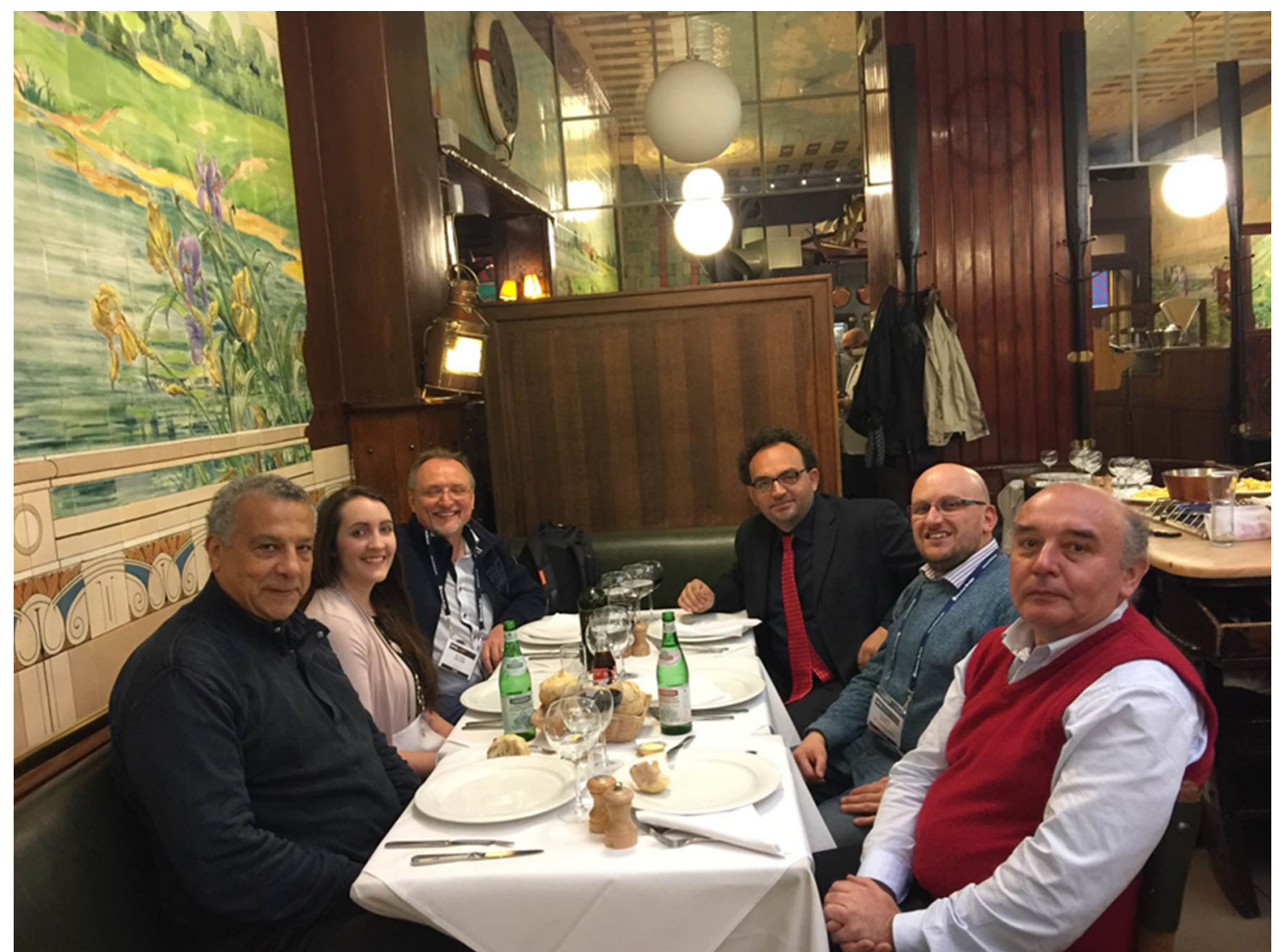

Fig. 1 (From left to right) Mohamed Akil, Rachel Moriarty, Matthias Carlsohn, Sergio Saponara, Guillermo Botella, and Bogdan Smolka

Concerning the last discussion topic, in order to cope with the increasing number of submissions and to bring new expertise to the editorial board, the following five colleagues have agreed to join the board: Tolga Birdal, Ricardo Carmona Galán, Dominique Ginhac, Byung-Gyu Kim, and Zoran Zivkovic. We welcome them to the editorial board. These colleagues have either previously conducted special issues as guest editors or been identified by the JRTIP reviewer reward program as very active and experienced reviewers.

This first issue of volume 12 comprises a total of 15 papers. The following three major themes are noted in these papers: (i) real-time GPU or FPGA implementations of image processing algorithms (six papers), (ii) improving computational efficiency or power efficiency of video coding (three papers), and (iv) computationally efficient image processing systems or algorithms (six papers). Brief summaries of these papers are outlined below which are ordered by these themes.

The first paper by Pang et al. is entitled "Fast Gabor texture feature extraction with separable filters using GPU." This paper proposes a GPU acceleration of the texture feature extraction algorithm by using separable 1D Gabor filters to approximate the non-separable Gabor filter kernels. Experimental results show that this approach improves the timing significantly with minimal error. This approach is then optimized to achieve a speed of $16 \mathrm{fps}$ on modest graphics hardware for an image size of 2562 pixels and a filter kernel of 322 pixels.

The second paper by Lamas-Rodríguez et al. is entitled "GPU-accelerated level-set segmentation." This paper presents two GPU implementations of a level-set-based segmentation method called "Fast Two-Cycle." Computations are carried out in independent domains and the structure of the original algorithm is matched to the GPU architecture. The implementations are tested using real images of brain vessels and a synthetic MRI brain image. The results obtained show that these implementations are faster than CPU implementations without any significant change in the segmentation quality.

The third paper by Perrot et al. is entitled "Fast GPUbased denoising filter using isoline levels." This paper describes a GPU-based filter for image denoising using the Matheron's level sets theory. A priori knowledge of the corrupting noise and the polygonal level-line modeling constraint are assumed in order to estimate the gray-level of the denoised image pixels by using local maximum likelihood optimization. It is shown that over $512 \times 512$ 
test images, the implementation done provides a high quality/runtime ratio in $9 \mathrm{~ms}$, leading to a processing rate of $19 \mathrm{fps}$.

The fourth paper by Islam et al. is entitled "GPU accelerated regional lung air volume measurements from phase contrast X-ray images." This paper discusses image processing techniques to measure regional lung air volume from phase contrast X-ray images with the objective of safely ventilating the lungs of preterm infants. The GPU platform is used to accelerate the processing of the image processing techniques involved. The performance of two different GPUs is examined, and their outcomes are compared with two CPUs. An overall speedup of 1.75-14.12 is attained for the absorption contrast-based computation technique while an overall speedup of $2.43-15.78$ is attained for the bound phase retrieval computation technique. It is also shown that the GPU-based lung volume measurements are in agreement with the CPU-based measurements.

The fifth paper by Chong et al. is entitled "Accelerated catadioptric omnidirectional view image unwrapping processing using GPU parallelization." This paper exploits the parallelism of graphical processing units (GPUs) involving the Compute Unified Device Architecture (CUDA) to provide an acceleration of the computationally intensive unwrapping component of distortion correction. A series of adaptations to CUDA is performed to enable an optimized usage of the hardware platform. The performance of the unwrapping is evaluated on a high-end and a low-end GPU to demonstrate the effectiveness of the parallelization performed.

The sixth paper by Kurdthongmee is entitled "A hardware centric algorithm for the best matching unit searching stage of the SOM-based quantizer and its FPGA implementation." This paper presents a hardware centric algorithm with the objective of accelerating the best matching unit (BMU) searching stage of the self-organizing-map or SOM-based quantizer. The algorithm relies on using the distance of a processing element as a memory address to store its index. The algorithm is designed to make it feasible for realization on an FPGA platform. The synthesis results compared with the conventional BMU searching indicate the algorithm runs 1.8 times faster while its FPGA resource requirements are higher than the conventional algorithms in terms of slices and look-up-table (LUT) usages.

The seventh paper by Cervero et al. is entitled "A scalable H.264/AVC deblocking filter architecture." This paper addresses the deblocking filter (DF) in the compression standards H.264/AVC and SVC by proposing a scalable DF architecture that is able to adapt its structure and performance to different video configurations. Its scalability allows one to avoid redesigning the architecture as the configuration settings change. In addition, the proposed architecture relies on a parallelization strategy to reduce the amount of clock cycles for filtering a video frame. An implementation of the architecture is done on the Virtex-5 FPGA, and the results obtained demonstrate its benefits.

The eighth paper by Correa et al. is entitled "Complexity scalability for real-time HEVC encoders." This paper addresses the problem of enabling complexity scalability in HEVC encoders by establishing a trade-off between processing time and rate-distortion (R-D) in a controlled manner. This trade-off is achieved based on dynamic constraining of HEVC coding tree blocks (CTBs) by limiting the prediction block shapes and the maximum tree depth used in each CTB. The developed complexityscalable encoder is capable of adjusting the processing time according to a predefined value. The results show that processing times can be scaled down to $50 \%$ with negligible R-D performance losses and to $20 \%$ with a maximum performance loss of $1.41 \mathrm{~dB}$, which is acceptable in many applications.

The ninth paper by Bilavarn et al. is entitled "Effectiveness of power strategies for video applications: a practical study." This paper examines the practical effectiveness of power strategies for video applications. Based on actual implementations of three power strategies, an analysis of application level parameters affecting the operability and efficiency of power strategies is carried out. The results obtained are found to be highly variable and sometimes exhibit power increases depending on the characteristics of the platform. Useful guidelines for successful power management are provided showing the benefits of the power strategies.

The tenth paper by Zhang et al. is entitled "Automatic reading of domestic electric meter: an intelligent device based on image processing and ZigBee/Ethernet communication." This paper covers an automatic reading system of household meters based on image processing. The meter reading is achieved in an accurate way by carrying out a number of image processing operations. The field testing of the system has led to a recognition rate of $99.7 \%$ with the identification time staying below $2 \mathrm{~s}$ with the outcome transmitted reliably through 1-3 walls in typical buildings.

The eleventh paper by Correa-Tome et al. is entitled "Fast similarity metric for real-time template-matching applications." This paper presents a visual similarity metric, named maximum cardinality similarity metric, based on precision-recall graphs as an alternative to the Hausdorff distance (HD). This metric is then used to establish similarity between a reference shape and a test shape. The matching problem is solved using the Hopcroft-Karp algorithm having low computational complexity. The results obtained show that the proposed methodology is 
more accurate under noisy conditions while running at lower processing times.

The twelfth paper by Chen et al. is entitled "Real-time human action recognition based on depth motion maps." This paper presents a human action recognition method by using depth motion maps (DMMs). DMMs are regarded as features which correspond to a depth video sequence projected onto three orthogonal Cartesian planes. These features are fed into a collaborative representation classifier to perform action recognition. The developed method is shown to be computationally efficient allowing it to run in real-time on a personal computer. The results obtained based on the Microsoft Research Action3D dataset indicate a performance superior to existing methods.

The thirteenth paper by Liu et al. is entitled "A study of hardware-friendly methods for gradient domain tone mapping of high dynamic range images." A large class of techniques for image processing is based on the manipulation of image gradient. These techniques normally have high computational complexity due to the need to solve an inverse problem. A family of methods, inspired by Fattal's operator, but with significant lower computational complexity, solves the inverse problem in small-size moving windows. In this paper, a study is conducted to understand the role that boundary conditions and window size play in the quality of resulting high dynamic range images.

The fourteenth paper by Hsia et al. is entitled "Directional prediction CamShift algorithm based on adaptive search pattern for moving object tracking." This paper covers a new CamShift algorithm, named Directional
Prediction CamShift (DP-CamShift), to improve the accuracy rate of tracking. An adaptive search pattern (ASP) is used to refine the searching of central areas. As a result, the process is made more robust by detecting the most likely direction of the moving target. For test video sequences of size $320 \times 240$, the experimental results indicate that the developed algorithm has an accuracy rate of $99 \%$ while achieving a processing rate of $23 \mathrm{fps}$.

The final fifteenth paper by Ramakrishnan et al. is entitled "Enhanced low-complexity pruning for corner detection." This paper introduces a two-step computationally efficient technique to accelerate the Shi-Tomasi and Harris corner detectors. The first step involves replacing the complex corner measure with simple approximations to quickly prune away non-corners. The second step involves applying the complex corner measure to a small corner candidate set obtained after pruning. The results obtained show that the proposed technique achieves up to $75 \%$ speedup on the hardware platform Nios-II, while generating corners with comparable or better accuracy than the conventional Shi-Tomasi and Harris detectors.

Finally, it is worth mentioning that Springer has made the article entitled "Overview and evaluation of the JPEG XT HDR image compression standard" available for free download till July 15 from this link http://link.springer. com/article/10.1007/s11554-015-0547-x, since this article has been identified as a high-impact article due to its potential for wider dissemination and exchange of HDR images supported by the JPEG XT standard. 